Symposium on evidence based medicine

\section{Ethics, philosophy, and evidence based medicine}

\section{R Ashcroft, $\mathbf{R}$ ter Meulen}

\section{The editors of the symposium hope it will provide a balanced} appraisal of evidence based medicine.

T his symposium is devoted to evidence based medicine (EBM) and the ethical issues it raises. Since Sir Archie Cochrane's seminal Nuffield Provincial Hospitals Trust lectures in 1972 and their publication as the Rock Carling monograph for that year, Effectiveness and Efficiency: Random Reflections on Health Services, the idea that medical interventions and health services should be evaluated and selected on the basis of the most reliable evidence available for their effectiveness and cost effectiveness has become very widely accepted. ${ }^{12}$ This widespread acceptance has not been complete, and it sometimes seems that there are as many critics of EBM as advocates. Whereas the importance of randomised trial evidence, critical appraisal, metaanalysis, and systematic review cannot be overestimated, the extent to which they comprise a panacea is open to question. The questions of how far EBM is able to answer questions of policy and value, and how far EBM is itself a value laden project, remain hotly debated. How can rational and ethical medical care and health policy be made in the absence of "perfect" evidence? How can we incorporate patient or community views into decision making? When does the search for evidence lead to problems of research ethics? How well founded is the claim that "evidence" gives us knowledge in the first place?

The papers in this issue were prepared under the auspices of the EVIBASE project. EVIBASE was a three year collaborative project funded by the European Commission to examine the ethical and philosophical issues underlying the theory and practice of EBM. The project involved theoretical research and empirical inquiry, with the latter concentrating on a series of country reports on the status of EBM in different European countries, the United States of America, and Australia, and interviews with key individuals involved in health policy, health services, medical research, and the Cochrane Collaboration. Some earlier results from the project have been published elsewhere. ${ }^{3}$ Those earlier as a tool in health policy and health systems reform. The present papers concentrate on medical practice and research.

The papers by Lie, Vineis, and Ashcroft examine the foundations of EBM in clinical research. Ashcroft and Vineis consider the logical, epistemological, and metaphysical foundations of clinical research evidence, wherease Lie considers how far EBM and health policy depend upon the randomised controlled trial, and how far trials are useful in making resource allocation decisions. The issue of the relationship between EBM and health care management is taken up by Biller-Andorno and colleagues, and by Rogers. BillerAndorno discusses the relationship treatment, whereas Rogers looks more widely at its implications for justice. Similar issues are taken up by papers concentrated on the role of EBM between EBM and decisions to fund
Berghmans and colleagues in their contribution to the final group of papers, on ethics and EBM in the case of cases often thought to present difficulties for evidence based approaches. Berghmans and colleagues discuss the case of psychiatry; Slowther and colleagues look at general practice; Ernst and colleagues discuss complementary and alternative medicine; Stirrat considers surgery; and Vos and colleagues examine the case of "orphaned fields of medicine".

The message of many of the papers collected here is of moderated scepticism. There is scepticism because there are serious philosophical and analytical problems yet to be overcome in delivering on the promise of EBM. There is moderated scepticism because EBM offers tools and concepts which do offer practical approaches to the challenge of a rational practice of medicine and health policy which are superior to most of the available alternative approaches. To question the foundations of a discipline or a practice is not necessarily to deny its value, but rather to stimulate a judicious and balanced appraisal of its merits; we offer the present selection of papers in that spirit.

J Med Ethics 2004;30:119.

doi: 10.1136/jme.2003.007286

.

Authors' affiliations

R E Ashcroft, Medical Ethics Unit, Imperial College, London, UK

$\mathbf{R}$ ter Meulen, Institute for Health Care Ethics, University of Maastricht, the Netherlands

Correspondence to: R E Ashcroft, Imperial College London, Department of Primary Health Care and General Practice, Reynolds Building, St Dunstan's Road, London W6 8RP, UK; r.ashcroft@imperial.ac.uk

\section{REFERENCES}

1 Cochrane AL. Effectiveness and efficiency: random reflections on health services. London: Nuffield Provincial Hospitals Trust, 1972.

2 Maynard A, Chalmers I, eds. Non-random reflections on health services research. London: BMJ Books, 1997.

3 Ter Meulen R, Dickenson D, eds. Into the hidden world of evidence based medicine. Health Care Analysis 2002;10:231-337. 


\section{Introduction to the symposium: what evidence based medicine is and what it is not}

\section{A Liberati, P Vineis}

\section{Evidence based medicine has much to offer, but a great deal remains to be done to create a better understanding of what it can and cannot do.}

T he term EBM (evidence based medicine), as we use it nowadays, was introduced in 1992 by the same group of people who, years before, founded the discipline called "Clinical epidemiology" (CE). ${ }^{1} \quad$ CE stemmed essentially from the idea of adapting and expanding epidemiological methods to medical and health care decision making; CE was in fact defined as " the discipline dealing with the study of the occurrence of medical decisions in relation to their determinants". ${ }^{1}$

CE has been very successful in illustrating new ways of teaching medicine and training health professionals, and has positioned itself around the notion that "critical appraisal skills" are yet another set of essential abilities whichin addition to the interpersonal, diagnostic, and prognostic skills-a good doctor should master. An important by product of CE was documentation which showed that much of the available evidence on diagnosis, prognosis, and treatment of diseases was of poor methodological quality and quite often of dubious transferability to everyday clinical practice.

This led to a strong call for improving the scientific basis of clinical practice, which was seen as too often dominated by practices of unproven effectiveness. This was the background for the 1992 Journal of the American Medical Association article that first used the term "evidence based medicine". ${ }^{2}$

In essence, proponents of EBM said that "all medical action of diagnosis, prognosis, and therapy should rely on solid quantitative evidence based on the best of clinical epidemiological research". ${ }^{2}$ Also they stated that "we should be cautious about actions that are only based on experience or extrapolation from basic science". ${ }^{2}$ Indeed, this is not a new concept, as recent research into the history of medicine has documented. ${ }^{3}$ Vandenbroucke recently discussed the well rooted historical precedents for the CE and EBM movements in the history of methodological research in medicine quoting, among others, Alexandre Louis who led an initiative called "médecine d'observation" in France in 1830. Finding, not surprisingly, strong resistance from his fellow physicians, Louis stated that "physicians should not rely on speculation and theory about causes of disease, nor on single experiences, but they should make large series of observations and derive numerical summaries from which real truth about the actual treatment of patients will emerge". ${ }^{3}$ Parallels and differences between now and then are worth noting here. In the early 1800 s proponents, of "médecine d'observation" were reacting against a kind of medicine that derived its theories from many things that we would consider "nonsense" by today's scientific standards. Today EBM acts in the context of a very different environment, in which modern medical basic science has a solid experimental background. We now know that "médecine d'observation," failed shortly after its appearance. A strong reaction from the medical profession together with the absence of contextual conditions account for this unfavourable outcome. Will EBM experience a different outcome as it exists in a more scientifically oriented medical world? In many ways a similarly strong negative reaction has emerged today against EBM. No doubt one of the reasons for such a negative reaction against EBM has been the fact that it was labelled as a "shift in medical paradigm". Such a definition would imply that EBM means scientific medicine and that all medicine practised before it was unscientific. This is not only simplistic but, as any closer scrutiny will reveal, profoundly wrong. The difference that needs to be marked is not that before EBM people did not use the evidence. Rather, the real failure was the lack of a framework and a set of rules to use the evidence in a systematic and explicit fashion.

Seen in this way the current fight around EBM and its nature could be advanced by moving the discussion from principles to a more pragmatic perspective, where the attention is centred on a "better use of evidence in medicine". This would have the distinct advantage of indicating that it is the way in which, and the rules according to which, we use and interpret evidence that need to be changed.

In contrast to the traditional wisdom of clinical practice, stressing the need for "a better use of evidence in medicine" would indicate that intuition and unsystematic clinical experience as well as a pathophysiological rationale are insufficient grounds for clinical decision making. On the contrary, the modern practice of medicine finds its way by reliance on formal rules aimed at interpreting the results of clinical research effectively; these rules must complement the medical training and common sense of clinicians, whose uncontrolled dominance is no longer ethically and scientifically acceptable.

Struggling for a better use of evidence in medicine also has other important advantages. It challenges the paternalistic and authoritarian nature of much medical practice and helps increase awareness that-even when based on scientific methods-there is a selective and structural imbalance in the nature of the evidence that is available, because that evidence is skewed and biased toward therapeutic $v$ preventive interventions and toward simple pharmacological $v$ complex behavioural/social care. Acquiring critical appraisal skills-one of the most important tenets of the EBM movement-is the necessary (though not sufficient) and best immunisation against ignoring that there is a structural imbalance in the research agenda; an imbalance that should be overcome in order to make fully available the sort of evidence that is needed to provide effective and comprehensive health care to all patients.

\section{PERSPECTIVES}

There is no doubt that EBM does not, and cannot, answer all the epistemological and practical questions surrounding the practice of medicine. On the contrary, it is important that expectations of EBM are appropriate in order to prevent conceptual and practical mistakes. Evidence based medicine provides methodological tools and a cultural framework. Methodologically it is useful to understand how we can produce valid and relevant information about the effectiveness of medical care. 


\section{GUEST EDITORIAL}

Culturally, its anti-authoritarian spirit is important in increasing the participation of different stakeholders and the opportunity for a multidisciplinary approach to health care problems.

It is clear that, thus far, the potential of EBM has not been fully exploited and that views of it which are too narrow have created avoidable confrontations with those who may be concerned that an "EBM dominated view" can do more harm than good. As efforts by methodologists have chiefly focused on how to design, conduct, and interpret studies aimed at assessing the efficacy/effectiveness of drugs, EBM is today mostly "evidence based therapy" with robust tools-that is, randomised controlled trials, especially for assessing the worth of relatively simple interventions. The fact that we currently have limited ability to reliably assess complex interventions, preventive care in general, and diagnosis as well as prognosis, should be seen not only as the result of the greater intrinsic complexity of these areas but also as the consequence of lower intellectual investments. This is a reflection, in turn, of the more limited commercial interests at stake here.

It is our view that-despite the many limitations we have highlighted in this paper-EBM has, at least in some areas of medicine, resulted in better clinical research and greater awareness of health professionals, health administrators, and policy makers of the need for medicine that is based on evidence. A lot remains to be done in order to create a better understanding of the nature of proof, evidence, and uncertainty; a more balanced research agenda; more coherent mechanisms to improve quality of care; and more substantial cultural efforts to empower patients and consumers. We should be ready, however, to recognise that most of this goes beyond what EBM can do alone and depends, more broadly, on health policy and politics with a capital " $\mathrm{P}$ ".
J Med Ethics 2004;30:120-121.

doi: 10.1136/jme.2003.007195

\section{Authors' affiliations}

A Liberati, University of Modena and Reggio

Emilia and Italian Cochrane Centre, Mario

Negri Institute Milano, Italy

P Vineis, University of Torino, ISI Foundation, Torino, Italy

Correspondence to: P Vineis, University of Torino, ISI Foundation, Torino, Italy; paolo.vineis@unito.it

\section{REFERENCES} 1969:89:125-8

2 Evidence Based Medicine Working Group Evidence based medicine: a new approach to the teaching of medicine. JAMA 1992;268:2420-5.

3 Vandenbroucke IP. Evidence based medicine and "médecine d'observation". J Clin Epidem 1996;49:1335-8.

4 Anon. Evidence based medicine: in its place Lancet 1995;346:1171-2.
1 Sackett DL. Clinical epidemiology. Am J Epidemiol 